\title{
Reported autism diagnosis strongly predicts psychotic-like experiences in the Adolescent Brain Cognitive Development cohort
}

\author{
Amandeep Jutla ${ }^{1,2}$, \\ Meghan Rose Donohue ${ }^{3}$, \\ Jeremy Veenstra-VanderWeele ${ }^{1,2,4}$, and \\ Jennifer H. Foss-Feig ${ }^{5}$ \\ ${ }^{1}$ Department of Psychiatry, Columbia University \\ ${ }^{2}$ New York State Psychiatric Institute \\ ${ }^{3}$ Department of Psychiatry, Washington University School of Medicine \\ ${ }^{4}$ Center for Autism and the Developing Brain, New York-Presbyterian Hospital \\ ${ }^{5}$ Seaver Autism Center for Research and Treatment, Icahn School of Medicine at Mount Sinai
}

\begin{abstract}
Although the schizophrenia rate is increased in autism spectrum disorder (ASD), it is difficult to identify which youth with ASD will go on to develop psychosis. We explored the relationship between $\mathrm{ASD}$ and emerging psychotic-like experiences in the Adolescent Brain Cognitive Development (ABCD) cohort of school-aged children. We predicted that ASD would robustly predict psychotic-like experience severity, even relative to other established predictors, and that ASD youth with psychotic-like experiences would have a characteristic neurocognitive profile. In a sample of 9,130 youth aged 9-11, we fit regression models that included parent-reported ASD, family history of psychosis, lifetime trauma, executive function, processing speed, working memory, age, sex, race, ethnicity, and income-to-needs ratio as predictors of Prodromal Questionnaire - Brief Child (PQ-BC) score. We assessed cognitive profiles using analysis of variance on NIH Toolbox measures. ASD increased PQ-BC distress scores by 2.46 points $(95 \%$ CI $1.32-3.60)$, an effect at least as large as those of family history of psychosis ( 1.05 points, $95 \%$ CI $0.56-1.53)$, Latinx ethnicity (0.99 points, $95 \%$ CI $0.52-1.45)$ and black race $(0.89$ points, $95 \%$ CI $0.30-1.48)$. We did not identify a unique cognitive profile for ASD youth with psychotic-like experiences. Our finding that ASD predicts psychotic-like symptoms in youth is consistent with previous literature, and adds new information in suggesting that ASD is a strong predictor of psychotic-like experiences even when compared to other established SCZ risk factors.
\end{abstract}

\section{Introduction}

Autism spectrum disorder (ASD) and schizophrenia (SCZ) are separate diagnostic entities that in some respects show clear divergence. ASD, typically diagnosed in childhood, is characterized by the combined presence of social communication deficits and restricted or repetitive behaviors ${ }^{1}$ and 
tends either to have a stable course or to show clinical improvement with time ${ }^{2}$. SCZ, typically diagnosed in late adolescence or early adulthood ${ }^{3}$, is characterized by psychosis, which reflects a loss of contact with reality, and manifests as delusional beliefs or hallucinatory experiences ${ }^{4}$. Unlike $\mathrm{ASD}$, it tends to be associated with progressive functional and cognitive decline ${ }^{5}$.

Despite their clear differences, ASD and SCZ also have notable similarities. Both have an impact on social skills, with impaired social communication a core deficit in ASD and an important cause of long-term disability in $\mathrm{SCZ}^{6}$. Both are characterized by deficits in non-social cognition, sharing impairments in executive function ${ }^{7,8}$, processing speed ${ }^{9,10}$ and working memory ${ }^{11,12}$.

Consistent with this, genome-wide association data demonstrate robust shared risk between ASD and $\mathrm{SCZ}^{13}$. Recent meta-analytic findings suggest that ASD diagnosis is in fact a SCZ risk factor. Individuals with ASD appear to be 3 to 4 times more likely to develop SCZ than members of the general population ${ }^{14,15}$.

Whether ASD is a more general risk factor for childhood psychotic-like experiences, in addition to a specific risk factor for SCZ diagnosis, remains an open question. "Psychotic-like experiences" constitute psychotic symptoms such as delusions or hallucinations in the absence of a frank psychotic disorder ${ }^{16}$, and in children may have prognostic value. For example, early psychotic-like experiences at age 11 increase the risk of schizophreniform disorder, a precursor to SCZ, at age $26^{17}$. In recent years, as SCZ has increasingly been viewed through a neurodevelopmental lens ${ }^{3}$, psychotic-like experiences have become a focus of research in their own right, as they may present an opportunity for intervention before clinical SCZ is diagnosed ${ }^{18}$.

When psychotic-like experiences persist and are associated with distress or impairment, they are thought to represent a meaningful diagnostic entity known as the "clinical high-risk for psychosis (CHR) syndrome," sometimes referred to as "attenuated" or "prodromal" psychosis ${ }^{19}$. Even though the CHR syndrome progresses to frank SCZ in just one-fourth of cases within 36 months of its identification ${ }^{20}$, CHR youth who do not progress continue to have pronounced social and cognitive impairment ${ }^{21}$ and may benefit from specialized attention.

Recent findings suggest that ASD youth with CHR show a symptom pattern and rate of conversion to psychosis similar to the general CHR population, yet may be under-referred to CHR clinical settings ${ }^{22}$. This may in part stem from the dearth of research exploring the nature and extent of the relationship between ASD and early psychotic-like experiences.

In particular, the effect size of ASD relative to other factors thought to predict SCZ or psychotic-like experiences, such as family history of psychosis ${ }^{23,24}$, childhood trauma ${ }^{25}$, or race ${ }^{26,27}$, is unknown. It is also unclear whether ASD children with psychotic-like experiences show a pattern of impairment in executive function, working memory and processing speed dissociable from the patterns seen in those with ASD alone or psychotic-like experiences alone in this age group.

We sought to address these gaps in knowledge by investigating ASD and psychotic-like experiences in a large national sample of 9- to 11-year-olds. We hypothesized that (1) an ASD diagnosis would predict severity of psychotic-like experiences, and (2) the profile of executive function, working memory, and processing speed impairment would differ among ASD children with psychotic-like experiences, ASD children without psychotic-like experiences, and children with psychotic-like experiences but not ASD. 


\section{Method}

\subsection{Study sample}

Our study drew from the Adolescent Brain Cognitive Development (ABCD; RRID:SCR_015769) study cohort. Detailed descriptions of the ABCD study's design and recruitment strategy have been published elsewhere ${ }^{28,29}$, but in brief, the study recruited 11,875 children from 21 sites across the United States. Recruitment used probability sampling of schools, with a goal of obtaining a representative cross-section of U.S. youth aged 9 to 11 to follow longitudinally over ten years into early adulthood. The ABCD study was approved by the institutional review board at each participating site.

Our study used baseline cross-sectional data collected from each participant between September 2016 and October 2018. Given our aims, we excluded 2,745 ABCD participants who were missing data for any of the following variables: ASD diagnosis, psychotic-like experience rating, family history of psychosis, age-adjusted standard scores for NIH toolbox measures of cognition, trauma history, age, sex, race, ethnicity, household income category, number of people in the household, history of speech delay, maternal age at birth, and paternal age at birth (see Table $\mathbf{S} 1$ for missingness by variable). This yielded a sample of 9,130 youth.

\subsection{Assessment measures}

ASD: As the ABCD study did not collect ASD-specific measures at baseline, we ascertained ASD based on whether a participant's parent(s) reported an existing ASD diagnosis during the screening interview. Note that we interrogated the validity of this ascertainment method by estimating a logistic regression model, ancillary to our main analysis, in which we examined whether variables known to be predictive of an ASD diagnosis (including speech delay, male sex, and older parental age) predicted parent report of ASD diagnosis during screening. Model coefficients are reported in the results section below.

Psychotic-like experiences: We identified psychotic-like experiences using the Prodromal Questionnaire - Brief Child version (PQ-BC). The PQ-BC is an adapted version of the Prodromal Questionnaire - Brief (PQ-B), a 21-item screening questionnaire for psychotic-like experiences in adolescents and adults. Each PQ-B item asks about the presence of a psychotic-like experience and then has the respondent rate, on a five-point scale, how much distress the symptom causes if present. The PQ-BC retains the PQ-B's 21 items, but with simplified, child-appropriate wording. To ensure that children understand the questions posed, these items are administered as an interview rather than a questionnaire, with the distress scale paired with a visual response analog 18,30 .

The PQ-BC, like the PQ-B, is scored by deriving two indices. For the "total" score, ranging from 0 to 21 , one point is assigned per symptom endorsed. For the "distress" score, ranging from 0 to 105, 1 to 5 points are assigned per symptom endorsed, based on a distress rating (where 1 indicates "no distress" and 5 "severe distress") ${ }^{18,30}$. As psychotic-like experiences are thought to be more predictive of CHR when associated with distress ${ }^{31}$ our study focused on the PQ-BC distress score.

We analyzed the distress score in two ways: 1) by using the score itself as a continuous indicator of psychotic-like experience severity, and 2) by using an empirical cutoff of a score 2 standard deviations above the mean to represent the binary presence of "likely significant" psychotic-like 
experiences. We used this cutoff because no studies have yet recommended a meaningful PQ$\mathrm{BC}$ cutoff, and the applicability of recommendations from previous studies using the PQ-B as a screening tool ${ }^{30,31}$ to a younger population is not clear.

Family history of psychosis: We defined family history of psychosis as a response of "yes" to the question "Has any blood relative of your child ever had a period lasting six months when they saw visions or heard voices or thought people were spying on them or plotting against them?"

Cognitive measures: The ABCD cognitive assessment ${ }^{32}$ included three NIH Toolbox Cognition Battery instruments relevant to our study: Dimensional Change Card Sort (RRID:SCR_003616), ${ }^{33,34}$, Pattern Comparison Processing Speed (RRID:SCR_003623) ${ }^{35}$, and List Sorting Working Memory (RRID:SCR_003626) [tulskyNIHToolboxCognition2013]. These are continuous measures of executive function, processing speed, and working memory respectively. All three have shown adequate convergent and discriminant validity, as well as good reliability in children, with reported test-retest intraclass correlation coefficients of $0.86-0.95$ for Dimensional Change Card Sort ${ }^{34}, 0.75-0.90$ for Pattern Comparison Processing Speed ${ }^{35}$, and $0.78-0.91$ for List Sorting Working Memory ${ }^{36}$.

Trauma history: Each participant's parent(s) received the traumatic events section of the Kiddie Schedule for Affective Disorders and Schizophrenia Present and Lifetime version (K-SADS-PL) structured interview ${ }^{37}$. This entails a series of yes/no questions about whether the participant has ever had certain lifetime traumatic experiences. Using these data, we derived a continuous "trauma index" for each participant, representing the number of traumatic experiences endorsed.

Demographic variables: Of the key demographic variables available in the ABCD dataset ${ }^{38}$, we included age at evaluation and sex assigned at birth as reported. Small numbers of participants for racial groups other than white or black required us to create an "other" racial group for statistical analysis. This group included Chinese, Filipinx, Indian, Japanese, Korean, Vietnamese, Native American, Native Alaskan, Native Hawaiian, Guamanian, Samoan, "other Asian," and "other Pacific Islander" participants, as well as "other race," "unknown race," and multiracial participants. We included ethnicity as a binary variable (Latinx vs. non-Latinx) independent from race.

We derived an income-to-needs ratio for each participant by (1) transforming their reported household income category into the mean income for that category and (2) dividing this by the poverty threshold for that participant's family size in the year that participant was interviewed ${ }^{39}$.

Other variables: We used a reported history of speech delay (defined as a parent response of "later" to the question "Would you say his/her speech development was earlier, average, or later than most other children?"), maternal age at birth, and paternal age at birth in our ancillary analysis of ASD predictors.

\subsection{Statistical approach}

First, we compared participants with and without ASD across the variables described above. For continuous variables, we compared means with Welch's two-sided unequal variances $t$-test, and for categorical variables we compared frequency counts with Pearson's $\chi^{2}$. Within each of these sets, we used the Benjamini-Hochberg procedure to control the false discovery rate.

Next, to ascertain whether ASD diagnosis predicted psychotic-like experiences (hypothesis 1), we estimated two regression models. The first model was a linear regression against continuous PQ- 
BC distress score. The second was a binary logistic regression against a PQ-BC distress cutoff $\geq 2$ standard deviations above the mean. Both models had a multilevel ("mixed-effect") structure, with family unit and $\mathrm{ABCD}$ recruitment site as nested random effects ${ }^{40}$, and were fit by restricted maximum likelihood using bound optimization by quadratic approximation.

Both models included the following inputs as fixed effects: ASD diagnosis, family history of psychosis, trauma index, executive function, processing speed, working memory, age, sex, race, ethnicity, and income-to-needs ratio. We selected these inputs in advance based on their availability in the ABCD dataset and their relevance as factors influencing schizophrenia or psychotic-like experiences. We entered variables using a simultaneous forced-entry strategy ${ }^{41}$, verified model stability using variance inflation factors to rule out collinearity, and evaluated model significance using Satterthwaite's approximation.

For both models we transformed continuous inputs (i.e., trauma index, age, income-to-needs ratio, and measures of executive function, processing speed, or working memory) by dividing by two standard deviations, while leaving binary inputs untransformed. This scaling method has been recommended ${ }^{42,43}$ for situations where models include a mixture of continuous and binary inputs, because it allows regression coefficients to be directly compared when assessing relative effect sizes. This is because the difference between two standard-deviation changes for a continuous variable represents the change from a very low $(\sim 2$ nd percentile) to a very high $(\sim 98$ th percentile) value. Such a change is comparable to the difference between the two conditions ( 0 or 1$)$ of a binary variable $^{42}$.

As an additional exploratory analysis related to hypothesis 1, we looked for group differences in mean item-level PQ-BC distress scores between participants with and without ASD. We conducted multiple univariate analysis of variance (ANOVA) tests, again accounting for multiple testing via the Benjamini-Hochberg method.

To compare executive function, working memory, and processing speed among ASD children with psychotic-like experiences, ASD children without psychotic-like experiences, and children with psychotic-like experiences but not ASD (hypothesis 2), we derived three groups (hereafter referred to, for simplicity, as ASD+/PLS+, ASD+/PLS-, and ASD-/PLS+), again using the PQ-BC distress score cutoff of $\geq 2$ standard deviations above the mean to represent the presence of likely significant psychotic-like experiences. We compared the three groups along each measure using one-way ANOVA, with Tukey's post-hoc test to identify pairwise differences.

Last, to investigate the validity of our method of ASD ascertainment, we estimated a forced-entry logistic regression model with parent-reported ASD as the outcome. Sex, history of speech delay, maternal age, paternal age, race, ethnicity, age, and income-to-needs ratio were inputs. Given the limited number of ASD cases in our sample, we did not use a multilevel model here. Instead, we used generalized estimating equations to adjust for family unit ${ }^{44}$, and did not adjust for ABCD recruitment site.

For all analyses we set an a priori significance threshold of $\alpha=0.05$.

\subsection{Software and data}

We conducted analyses in R 3.5.1 $1^{45}$ using lme4 1.1-21 for modeling. 
Scripts to reproduce the results reported here are available from the authors at https://github. com/amandeepjutla/2019-abcd-asd.

The ABCD dataset is available to interested researchers through the NIMH Data Archive (https: //nda.nih.gov/). As the ABCD dataset grows and changes over time, individual data releases are assigned permanent digital object identifiers (DOIs). We used release 2.0.1, which can be found at http://dx.doi.org/10.15154/1504041.

In deriving income-to-needs ratios, we used annual poverty threshold data for 2016, 2017, and 2018 from the US Census Bureau (https://www.census.gov/).

\section{Results}

\subsection{Sample characteristics}

As shown in Table 1, the mean age of our sample's 9,130 participants was 9.91 years $(S D 0.62$, range 9.00 - 10.92). $4,778(52.33 \%)$ were male. Mean income-to-needs ratio was 4.51 (SD 2.88, range $0.10-16.02)$. Mean number of reported traumatic experiences (i.e., trauma index score) was $0.52(S D 1.08$, range $0-17) .957$ participants $(10.48 \%)$ had a parent-reported family history of psychosis.

Regarding race, 6,186 (67.75\%) were white, 1,185 (12.98\%) black, and 1,715 (18.78\%) "other." Of participants in the "other" category, $46(0.50 \%)$ were Chinese, $30(0.33 \%)$ Filipinx, $44(0.48 \%)$ Indian, 6 (0.07\%) Japanese, 9 (0.10\%) Korean, 7 (0.08\%) Vietnamese, $32(0.35 \%)$ Native American, 1 (0.01\%) Samoan, 18 (0.20\%) "other Asian," 8 (0.09\%) "other Pacific islander," 414 (4.53\%) "other race," 41 (0.45\%) "unknown race," and 1,141 (12.50\%) multiracial. 1,708 (18.71\%) participants were of Latinx ethnicity. Of Latinx participants, 971 (56.85\%) were white, 68 (3.98\%) were black, and $669(39.17 \%)$ were "other."

Mean PQ-BC total score was 2.56 ( $S D$ 3.51, range 0 - 20). Mean PQ-BC distress score was 3.50 $(S D 7.31$, range $0-84)$. $425(4.65 \%)$ participants had a PQ-BC distress score $\geq$ the 2 standard deviations above the mean threshold (which meant a score above 18). 151 participants (1.65\%) had a parent-reported ASD diagnosis.

Participants with ASD differed from those without it in several ways. They had higher PQ-BC total scores, $t(153.00)=-3.95, p=1.18 \times 10^{-3}$ and higher distress scores, $t(152.82)=-3.18$, $p=7.24 \times 10^{-3}$. Their executive function was relatively impaired, $t(154.14)=2.27, p=0.04$, as were processing speed, $t(154.66)=2.44, p=0.04$, and working memory, $t(154.04)=3.12, p=0.01$. They had older fathers, $t(154.82)=-2.29, p=0.04$ and were more likely to be male, $\chi^{2}=72.93$, $p=4.70 \times 10^{-17}$ ) or to have a history of speech delay, $\chi^{2}=201.01, p=8.82 \times 10^{-45}$.

\subsection{Hypothesis 1: ASD as predictor of psychotic-like experiences}

\subsubsection{Linear model: ASD as predictor of continuous PQ-BC distress score}

In the linear model, ASD had a strong positive effect on PQ-BC distress score (Table 2), with ASD youth having scores 2.46 points higher on average than non-ASD youth $(\beta=2.46,95 \%$ confidence interval (CI) $\left.1.32-3.60, t=4.23, p=2.31 \times 10^{-5}\right)$. 
This absolute effect size was at least as strong as those of other predictors in the model, including the positive predictor family history of psychosis, which increased PQ-BC distress scores by 1.05 points on average $\left(\beta=1.05,95 \%\right.$ CI $\left.0.56-1.53, t=4.20, p=2.76 \times 10^{-5}\right)$, the positive predictor Latinx ethnicity, which increased scores by an average of 0.99 points, $(\beta=0.99,95 \%$ CI $0.52-1.45$, $\left.t=4.19, p=2.86 \times 10^{-5}\right)$, the positive predictor black race, which increased scores by an average of 0.89 points $\left(\beta=0.89,95 \%\right.$ CI $\left.0.30-1.48, t=2.95, p=3.17 \times 10^{-3}\right)$, the negative predictor income-to-needs ratio, for which an increase of two standard deviations decreased PQ-BC distress scores by an average of 1.23 points $\left(\beta=-1.23,95 \%\right.$ CI $\left.-1.58--0.89, t=-7.02, p=2.34 \times 10^{-12}\right)$, and the negative predictor working memory skills, for which a two-standard deviation increase decreased PQ-BC distress scores by an average of 1.12 points $(\beta=-1.12,95 \%$ CI $-1.43--0.81$, $\left.t=-7.11, p=1.29 \times 10^{-12}\right)$.

The effect of ASD was also larger than the positive predictor trauma, for which an increase in trauma index score by two standard deviations raised PQ-BC scores by 0.55 points $(\beta=0.55,95 \%$ CI $0.26-0.85, t=3.68, p=2.30 \times 10^{-4}$ ) and the negative predictor age, for which an increse in two standard deviations reduced PQ-BC scores by an average of 0.75 points $(\beta=-0.75,95 \%$ CI $\left.-1.05--0.46, t=-5.01, p=5.43 \times 10^{-7}\right)$.

Sex, white race, executive functioning, and processing speed were not significant predictors in this model.

\subsubsection{Logistic model: ASD as predictor of PQ-BC score above a threshold}

The logistic model, in we regressed against $\mathrm{PQ}$-BC distress score $\geq 2$ standard deviations above the mean (Table 3) illustrates ASD's effect size in concrete terms. ASD predicted of PQ-BC score above this threshold (OR 3.18, 95\% CI $1.77-5.69, p=1.03 \times 10^{-4}$ ) with an effect at least as strong as those of Latinx ethnicity (OR 1.69, 95\% CI $1.26-2.26, p=4.92 \times 10^{-4}$ ), family history of psychosis (OR 1.68, 95\% CI 1.25 - 2.25, $p=6.07 \times 10^{-4}$ ), income-to-needs ratio (OR $0.49,95 \%$ CI $0.38-0.64, p=1.32 \times 10^{-7}$ ), and working memory (OR $0.51,95 \%$ CI $0.40-0.65$, $p=2.70 \times 10^{-8}$ ). The effect of ASD was also stronger than those of trauma (OR 1.20, 95\% CI $1.01-1.44, p=4.25 \times 10^{-2}$ ) and age (OR 0.75, 95\% CI $\left.0.60-0.94, p=1.22 \times 10^{-2}\right)$. Sex, white

race, black race, executive functioning, and processing speed were not significant predictors in this model.

\subsubsection{Exploratory analysis: group differences in item-level PQ-BC distress scores}

The results of univariate ANOVAs comparing mean item-level PQ-BC distress scores between participants with and without ASD are provided in Table S2. After Benjamini-Hochberg correction, individuals with ASD scored higher than those without on eleven items.

In ascending order of statistical significance, these were items \#14 ("Did you feel confused because something you experienced didn't seem real, or it seemed imaginary to you?", $\mathrm{F}(1,9,128=5.20$, $p=0.04)$ ), \#12 ("Did you start to worry at times that your mind was trying to trick you or was not working right?", $\mathrm{F}(1,9,128=6.27, p=0.03)$ ), \#20 ("Did you suddenly start to be able to see things that other people could not see, or they did not seem to see?", $\mathrm{F}(1,9,128=6.04$, $p=0.03)$ ), \#1 ("Did places that you know well, such as your bedroom, or other rooms in your home, your classroom, or school yard, suddenly seem weird, strange or confusing to you, like not the real world?", $\mathrm{F}(1,9,128=6.38, p=0.03)$ ), \#2 ("Did you hear strange sounds that you never 
noticed before, like banging, clicking, hissing, clapping, or ringing in your ears?", $\mathrm{F}(1,9,128=$ $7.30, p=0.02)$ ), \#10 ("Did you lose concentration because you noticed sounds in the distance that you usually don't hear?", $\mathrm{F}(1,9,128=8.00, p=0.02)$ ), \#17 ("Did you feel that sometimes your thoughts were strong you could almost hear them, as if another person, not you, spoke them?", $\mathrm{F}(1,9,128=7.21, p=0.02)$ ), \#18 ("Did you feel that other people might want something bad to happen to you or that you could not trust other people?", $\mathrm{F}(1,9,128=7.08, p=0.02)$ ), \#11 ("Although you could not see anything or anyone, did you suddenly start to feel that an invisible energy, creature, or some person was around you?", $\mathrm{F}(1,9,128=10.80, p=0.01)$ ), \#15 ("Did you honestly believe in things that other people would say are unusual or weird?", $\mathrm{F}(1,9,128=11.29$, $p=0.01)$ ), and \#21 ("Did you suddenly start to notice that people sometimes had a hard time understanding what you were saying, even though they used to understand you well?", F(1, 9,128 $\left.\left.=13.65, p=4.65 \times 10^{-3}\right)\right)$.

\subsection{Hypothesis 2: Different neurocognitive profiles among sub-groups}

Our results did not support hypothesis 2, that neurocognitive profiles would differ between $\mathrm{ASD}+/ \mathrm{PLS}+(\mathrm{n}=18), \mathrm{ASD}+/$ PLS- $(\mathrm{n}=133)$ and ASD-/PLS+ $(\mathrm{n}=407)$ participants. We did not identify significant differences between these groups along any of the three NIH Toolbox measures we had selected (executive function: $\mathrm{F}(2,555=1.28, p=0.28)$; processing speed: $\mathrm{F}(2$, $555=0.15, p=0.86)$; working memory: $\mathrm{F}(2,555=2.27, p=0.10)$.

\subsection{Robustness of findings}

We tested the robustness of our findings by addressing a notable limitation of the approach we took: namely, that our ascertainment of ASD was based on parent report, and not on gold-standard diagnosis by a clinician. This necessitated that we assume parent-reported ASD was a reasonably accurate proxy for "confirmed" ASD.

To examine this assumption, we estimated a logistic regression model with parent-reported ASD as the outcome and a series of predictors chosen based on their having a previously-described association with ASD and their availability in the ABCD dataset: male sex, history of speech delay, paternal age, and maternal age ${ }^{1}$. We also included race, ethnicity, age, and income-to-needs as covariates. The results of this regression, shown in Table S3, indicate that the predictors of parent-reported ASD in the ABCD cohort are consistent with known predictors of ASD in the general population, with history of speech delay (OR 6.09, 95\% CI $4.34-8.53, p<2 \times 10^{-16}$ ), male sex (OR 4.48, 95\% CI $2.77-7.25, p=1.06 \times 10^{-9}$ ) and paternal age (OR 1.64, 95\% CI $1.02-2.64$, $p=0.04)$ reaching statistical significance. We consider these results an indication that our use of parent-reported ASD in this study is reasonable.

\subsection{Sensitivity analyses}

In sensitivity analyses, we estimated alternate versions of the models used to test hypothesis 1 . We found that the pattern of our results was robust when we restricted our analysis to households with only one child participating in ABCD and when we used PQ-BC total score rather than distress score as our dependent variable. In both cases, ASD's effect remained at least as strong as those of other predictors in the model. 


\section{Discussion}

Our finding that ASD diagnosis predicts psychotic-like experiences in youth is consistent with literature suggesting that rates of SCZ and psychosis are greater in adults with ASD than in the general population ${ }^{14,15}$. It also adds new information in suggesting that the magnitude of ASD as a risk factor for psychotic-like experiences is significant even when compared to other established SCZ risk factors, such as family history and lower income-to-needs ratio.

Although it is possible that ASD is acting as a proxy for another SCZ risk factor we have not considered, we consider this unlikely, as it is unclear what this factor would be. In contrast, black race and Latinx ethnicity, which in our results also appeared to predict psychotic-like experiences, could have been proxies for other known SCZ/psychosis risk factors not included in our models, such as urbanicity, an ethnic density effect, or first- or second-generation immigration status ${ }^{46}$.

Our results therefore may have implications for both clinicians working primarily with ASD youth and clinicians working with CHR and early psychosis populations. Clinicians who see adolescents with ASD may benefit from incorporating psychosis screens into their assessments, and referring to CHR assessment clinics when indicated. By the same token, clinicians working with the CHR population might consider finding ways to connect with those working with the ASD population to ensure that a referral pipeline is in place.

Findings also are of import for researchers interested in the nosology of ASD and psychotic disorders. Our exploratory finding that ASD youth had higher mean distress scores on 11 of the PQ-BC's 21 items requires independent replication, but has interesting implications. Although some of these items may indicate individual psychotic-like pheomena of particular importance in the ASD population, some could simply reflect the sensory hypersensitivity, special or unusual interests, and communication difficulties that are common in $\mathrm{ASD}^{1}$. The possibility that some questions are indexing core ASD features rather than unique psychotic-like symptoms highlights the difficulty of identifying psychotic experiences in ASD. It suggests a possible need for adapted assessment instruments that capture the unique phenomenology of psychosis in this population.

We were not able to identify a cognitive profile specific to individuals having both reported ASD and psychotic-like experiences. This may in part be a function of sample size. Although the ABCD cohort is large, only 151 participants had reported ASD, and of those, 18 also had psychotic-like symptoms. Future studies that combine data collected from multiple large cohorts might have the power to detect subtle differences along cognitive dimensions. In the future, studies might also explore aspects of social cognition, language, and sensory functioning to better dissociate these groups.

We acknowledge that our study has some important limitations. Our method of ASD ascertainment - parent report of an ASD diagnosis - is less robust than a gold-standard diagnosis might have been. However, evidence suggests that parent-reported ASD is often accurate. In one study of 118 youth with parent-reported ASD enrolled in the nationwide Interactive Autism Network registry, parents were able to provide records confirming an ASD diagnosis in $116(98.31 \%)$ cases $^{47}$. In a similar study of enrollees in the Autism Spectrum Database - UK study, records substantiated parent-reported ASD in 142 of $156(91.03 \%)$ cases $^{48}$. Our finding that predictors of parent-reported ASD in the ABCD cohort are similar to known predictors of ASD lends further support to the idea that parent report is a meaningful indicator. 
Although ABCD is not an ASD-focused study, it is notable that even in this dataset, collected without an intention to examine ASD as a risk factor for psychotic-like experiences, we nevertheless found a strong relationship. In our view, this speaks to the robustness of this association. It also supports results from registry studies that similarly were not designed with an eye towards recruiting participants with ASD or psychotic symptoms ${ }^{49,50}$.

The ABCD cohort is relatively young, and most work examining convergence between ASD and schizophrenia has been conducted with older groups who have formal diagnoses. This limits the generalizability of our findings. However, our identification of an association between ASD and psychotic-like symptoms well before the psychosis prodrome typically appears raises intriguing possibilities for future research.

This is, to our knowledge, the first study to examine the association between ASD and psychotic-like experiences in middle childhood. As the ABCD cohort ages, and as longitudinal data is collected, we hope to delineate how ASD relates to trajectories of psychotic-like experiences over time, leading to a more robust understanding of how, when, and why ASD and SCZ converge.

\section{Acknowledgements}

This project was financially supported by a Whitaker Scholar in Developmental Neuropsychiatry Award to Dr. Jutla funded by the Marilyn and James Simons Foundation.

The ABCD study is supported by the National Institutes of Health and additional federal partners under award numbers U01DA041022, U01DA041028, U01DA041048, U01DA041089, U01DA041106, U01DA041117, U01DA041120, U01DA041134, U01DA041148, U01DA041156, U01DA041174, U24DA041123, and U24DA041147. Information about the study's supporters, participating sites, and study investigators can be found at https://abcdstudy.org. Although ABCD investigators provided data, they did not participate in the analysis or writing of this report. This manuscript therefore reflects the views of the authors and does not necessarily reflect the opinions or views of the NIH or ABCD consortium investigators.

\section{Conflict of Interest}

Dr. Veenstra-VanderWeele has consulted or served on an advisory board for Roche Pharmaceuticals, Novartis, and SynapDx; has received research funding from Roche Pharmaceuticals, Novartis, SynapDx, Seaside Therapeutics, and Forest; and has received an editorial stipend from Springer and Wiley. The remaining authors report no biomedical financial interests or potential conflicts of interest.

\section{References}

1. Lord C, Brugha TS, Charman T, et al. Autism spectrum disorder. Nat Rev Dis Primers. 2020;6(1):1-23. doi:10.1038/s41572-019-0138-4

2. Simonoff E, Kent R, Stringer D, et al. Trajectories in symptoms of autism and cognitive ability in autism from childhood to adult life: Findings from a longitudinal epidemiological cohort. Journal of the American Academy of Child \&3 Adolescent Psychiatry. 2019;0(0). doi:10.1016/j.jaac.2019.11.020 
3. Owen MJ, Sawa A, Mortensen PB. Schizophrenia. The Lancet. 2016;388(10039):86-97. doi:10.1016/S0140-6736(15)01121-6

4. Lieberman JA, First MB. Psychotic disorders. Ropper AH, ed. New England Journal of Medicine. 2018;379(3):270-280. doi:10.1056/NEJMra1801490

5. Fett A-KJ, Velthorst E, Reichenberg A, et al. Long-term changes in cognitive functioning in individuals with psychotic disorders: Findings from the Suffolk County Mental Health Project. JAMA Psychiatry. December 2019. doi:10.1001/jamapsychiatry.2019.3993

6. Green MF, Horan WP, Lee J. Nonsocial and social cognition in schizophrenia: Current evidence and future directions. World Psychiatry. 2019;18(2):146-161. doi:10.1002/wps.20624

7. Freedman D, Brown AS. The developmental course of executive functioning in schizophrenia. Int J Dev Neurosci. 2011;29(3):237-243. doi:10.1016/j.ijdevneu.2010.11.003

8. Demetriou EA, Lampit A, Quintana DS, et al. Autism spectrum disorders: A meta-analysis of executive function. Molecular Psychiatry. 2018;23(5):1198-1204. doi:10.1038/mp.2017.75

9. Knowles EE, David AS, Reichenberg A. Processing speed deficits in schizophrenia: Reexamining the evidence. AJP. 2010;167(7):828-835. doi:10.1176/appi.ajp.2010.09070937

10. Haigh SM, Walsh JA, Mazefsky CA, Minshew NJ, Eack SM. Processing speed is impaired in adults with autism spectrum disorder, and relates to social communication abilities. J Autism Dev Disord. 2018;48(8):2653-2662. doi:10.1007/s10803-018-3515-Z

11. Forbes NF, Carrick LA, McIntosh AM, Lawrie SM. Working memory in schizophrenia: A meta-analysis. Psychol Med. 2009;39(6):889-905. doi:10.1017/S0033291708004558

12. Wang Y, Zhang Y-b, Liu L-l, et al. A meta-analysis of working memory impairments in autism spectrum disorders. Neuropsychol Rev. 2017;27(1):46-61. doi:10.1007/s11065-016-9336-y

13. Consortium TB, Anttila V, Bulik-Sullivan B, et al. Analysis of shared heritability in common disorders of the brain. Science. 2018;360(6395):eaap8757. doi:10.1126/science.aap8757

14. Zheng Z, Zheng P, Zou X. Association between schizophrenia and autism spectrum disorder: A systematic review and meta-analysis. Autism Res. 2018;11(8):1110-1119. doi:10.1002/aur.1977

15. Lai M-C, Kassee C, Besney R, et al. Prevalence of co-occurring mental health diagnoses in the autism population: A systematic review and meta-analysis. Lancet Psychiatry. 2019;6(10):819-829. doi:10.1016/S2215-0366(19)30289-5

16. Kelleher I, Cannon M. Psychotic-like experiences in the general population: Characterizing a high-risk group for psychosis. Psychol Med. 2011;41(1):1-6. doi:10.1017/S0033291710001005

17. Poulton R, Caspi A, Moffitt TE, Cannon M, Murray R, Harrington H. Children's self-reported psychotic symptoms and adult schizophreniform disorder: A 15-year longitudinal study. Arch Gen Psychiatry. 2000;57(11):1053-1058. doi:10.1001/archpsyc.57.11.1053

18. Karcher NR, Barch DM, Avenevoli S, et al. Assessment of the Prodromal QuestionnaireBrief Child Version for measurement of self-reported psychoticlike experiences in childhood. JAMA Psychiatry. June 2018. doi:10.1001/jamapsychiatry.2018.1334 
19. Addington J, Stowkowy J, Weiser M. Screening tools for clinical high risk for psychosis. Early Intervention in Psychiatry. 2015;9(5):345-356. doi:10.1111/eip.12193

20. Pablo GS de, Catalan A, Fusar-Poli P. Clinical validity of DSM-5 attenuated psychosis syndrome: Advances in diagnosis, prognosis, and treatment. JAMA Psychiatry. November 2019. doi:10.1001/jamapsychiatry.2019.3561

21. Addington J, Stowkowy J, Liu L, et al. Clinical and functional characteristics of youth at clinical high-risk for psychosis who do not transition to psychosis. Psychological Medicine. 2018:18. doi:10.1017/S0033291718002258

22. Foss-Feig JH, Velthorst E, Smith L, et al. Clinical profiles and conversion rates among young individuals with autism spectrum disorder who present to clinical high risk for psychosis services. Journal of the American Academy of Child $\mathcal{E}$ Adolescent Psychiatry. February 2019. doi:10.1016/j.jaac.2018.09.446

23. Agerbo E, Sullivan PF, Vilhjálmsson BJ, et al. Polygenic risk score, parental socioeconomic status, family history of psychiatric disorders, and the risk for schizophrenia: A Danish population-based study and meta-analysis. JAMA Psychiatry. 2015;72(7):635-641. doi:10.1001/jamapsychiatry.2015.0346

24. Lu Y, Pouget JG, Andreassen OA, et al. Genetic risk scores and family history as predictors of schizophrenia in Nordic registers. Psychol Med. 2018;48(7):1201-1208. doi:10.1017/S0033291717002665

25. Morgan C, Gayer-Anderson C. Childhood adversities and psychosis: Evidence, challenges, implications. World Psychiatry. 2016;15(2):93-102. doi:10.1002/wps.20330

26. Bresnahan M, Begg MD, Brown A, et al. Race and risk of schizophrenia in a US birth cohort: Another example of health disparity? Int J Epidemiol. 2007;36(4):751-758. doi:10.1093/ije/dym041

27. Olbert CM, Nagendra A, Buck B. Meta-analysis of Black vs. White racial disparity in schizophrenia diagnosis in the United States: Do structured assessments attenuate racial disparities? J Abnorm Psychol. 2018;127(1):104-115. doi:10.1037/abn0000309

28. Volkow ND, Koob GF, Croyle RT, et al. The conception of the ABCD study: From substance use to a broad NIH collaboration. Developmental Cognitive Neuroscience. 2018;32:4-7. doi:10.1016/j.dcn.2017.10.002

29. Garavan H, Bartsch H, Conway K, et al. Recruiting the ABCD sample: Design considerations and procedures. Developmental Cognitive Neuroscience. 2018;32:16-22. doi:10.1016/j.den.2018.04.004

30. Loewy RL, Pearson R, Vinogradov S, Bearden CE, Cannon TD. Psychosis risk screening with the Prodromal Questionnaire - Brief Version (PQ-B). Schizophr Res. 2011;129(1):42-46. doi:10.1016/j.schres.2011.03.029

31. Kline E, Thompson E, Bussell K, Pitts SC, Reeves G, Schiffman J. Psychosis-like experiences and distress among adolescents using mental health services. Schizophrenia Research. 2014;152(23):498-502. doi:10.1016/j.schres.2013.12.012

32. Luciana M, Bjork JM, Nagel BJ, et al. Adolescent neurocognitive development and impacts of substance use: Overview of the adolescent brain cognitive development (ABCD) 
baseline neurocognition battery. Developmental Cognitive Neuroscience. 2018;32:67-79. doi:10.1016/j.den.2018.02.006

33. Zelazo PD. The Dimensional Change Card Sort (DCCS): A method of assessing executive function in children. Nature Protocols. 2006;1(1):297-301. doi:10.1038/nprot.2006.46

34. Zelazo PD, Anderson JE, Richler J, Wallner-Allen K, Beaumont JL, Weintraub S. NIH Toolbox Cognition Battery (CB): Measuring executive function and attention. Monographs of the Society for Research in Child Development. 2013;78(4):16-33. doi:10.1111/mono.12032

35. Carlozzi NE, Tulsky DS, Kail RV, Beaumont JL. NIH Toolbox Cognition Battery (CB): Measuring Processing Speed. Monographs of the Society for Research in Child Development. 2013;78(4):88102. doi: $10.1111 /$ mono. 12036

36. Tulsky DS, Carlozzi NE, Chevalier N, Espy KA, Beaumont JL, Mungas D. NIH Toolbox Cognition Battery (CB): Measuring working memory. Monographs of the Society for Research in Child Development. 2013;78(4):70-87. doi:10.1111/mono.12035

37. Kaufman J, Birmaher B, Brent D, et al. Schedule for Affective Disorders and Schizophrenia for School-Age Children-Present and Lifetime Version (K-SADS-PL): Initial reliability and validity data. Journal of the American Academy of Child $\mathcal{E}$ Adolescent Psychiatry. 1997;36(7):980-988. doi:10.1097/00004583-199707000-00021

38. Barch DM, Albaugh MD, Avenevoli S, et al. Demographic, physical and mental health assessments in the adolescent brain and cognitive development study: Rationale and description. Developmental Cognitive Neuroscience. 2018;32:55-66. doi:10.1016/j.dcn.2017.10.010

39. McLoyd VC. Socioeconomic disadvantage and child development. American Psychologist. 1998;53(2):185. doi:10.1037/0003-066X.53.2.185

40. Goldstein H, Browne W, Rasbash J. Multilevel modelling of medical data. Statistics in Medicine. 2002;21(21):3291-3315. doi:10.1002/sim.1264

41. Mundry R, Nunn CL. Stepwise model fitting and statistical inference: Turning noise into signal pollution. Am Nat. 2009;173(1):119-123. doi:10.1086/593303

42. Gelman A. Scaling regression inputs by dividing by two standard deviations. Statistics in Medicine. 2008;27(15):2865-2873. doi:10.1002/sim.3107

43. Schielzeth H. Simple means to improve the interpretability of regression coefficients. Methods in Ecology and Evolution. 2010;1(2):103-113. doi:10.1111/j.2041-210X.2010.00012.x

44. Hanley JA, Negassa A, Edwardes MD deB, Forrester JE. Statistical analysis of correlated data using generalized estimating equations: An orientation. Am J Epidemiol. 2003;157(4):364-375. doi:10.1093/aje/kwf215

45. Team RC. R: A Language and Environment for Statistical Computing. Vienna, Austria; 2018. http://www.r-project.org.

46. Radua J, Ramella-Cravaro V, Ioannidis JPA, et al. What causes psychosis? An umbrella review of risk and protective factors. World Psychiatry. 2018;17(1):49-66. doi:10.1002/wps.20490 
47. Daniels AM, Rosenberg RE, Anderson C, Law JK, Marvin AR, Law PA. Verification of parentreport of child autism spectrum disorder diagnosis to a web-based autism registry. J Autism Dev Disord. 2012;42(2):257-265. doi:10.1007/s10803-011-1236-7

48. Warnell F, George B, McConachie H, Johnson M, Hardy R, Parr JR. Designing and recruiting to UK autism spectrum disorder research databases: Do they include representative children with valid ASD diagnoses? BMJ Open. 2015;5(9). doi:10.1136/bmjopen-2015-008625

49. Selten J-P, Lundberg M, Rai D, Magnusson C. Risks for nonaffective psychotic disorder and bipolar disorder in young people with autism spectrum disorder: A population-based study. JAMA Psychiatry. 2015;72(5):483-489. doi:10.1001/jamapsychiatry.2014.3059

50. Schalbroeck R, Termorshuizen F, Visser E, Amelsvoort T van, Selten JP. Risk of non-affective psychotic disorder or bipolar disorder in autism spectrum disorder: A longitudinal register-based study in the Netherlands. Psychological Medicine. 2018:1-8. doi:10.1017/S0033291718003483

\section{Tables}

Table 1: Characteristics of study participants across ASD and non-ASD groups.

Table 2: Predictors of continuous PQ-BC distress score.

Table 3: Predictors of PQ-BC distress score above 2 SD cutoff.

\section{Supplementary Tables}

Table S1: Missingness by variable.

Table S2: Item-level PQ-BC distress score comparisons.

Table S3: Predictors of parent-reported ASD. 
medRxiv preprint doi: https://doi.org/10.1101/2020.02.07.20021170; this version posted February 11, 2020. The copyright holder for this preprint (which was not certified by peer review) is the author/funder, who has granted medRxiv a license to display the preprint in perpetuity.

It is made available under a CC-BY-NC-ND 4.0 International license .

Table 1

Characteristics of study participants across $A S D$ and non-ASD groups

\begin{tabular}{|c|c|c|c|c|}
\hline Variable & Ill sample $(n=9130)$ & No ASD $(n=8979)$ & $\operatorname{ASD}(n=151)$ & $p$ \\
\hline Continuous & $\underline{M(S D)}$ & $\underline{M(S D)}$ & $\underline{M(S D)}$ & $\underline{t \text {-test }}$ \\
\hline Age (in years) & $9.91(0.62)$ & $9.91(0.62)$ & $10.00(0.61)$ & 0.12 \\
\hline PQ-BC total score & $2.56(3.51)$ & 2.53 (3.49) & $4.00(4.54)$ & $1.18 \times 10^{-3^{\star \star}}$ \\
\hline PQ-BC distress score & $3.50(7.31)$ & $3.46(7.26)$ & $5.99(9.72)$ & $7.24 \times 10^{-3 \star \star}$ \\
\hline Income-to-needs ratio & $4.51(2.88)$ & $4.51(2.88)$ & $4.33(3.03)$ & 0.48 \\
\hline Trauma index score & $0.52(1.08)$ & $0.52(1.09)$ & $0.57(0.78)$ & 0.45 \\
\hline NIH TB Card Sort (executive function) & $97.38(15.25)$ & $97.43(15.22)$ & $94.29(16.86)$ & $0.04^{*}$ \\
\hline NIH TB Pattern Comparison (processing sp & $94.18(21.97)$ & $94.25(21.94)$ & $89.66(22.93)$ & $0.04^{*}$ \\
\hline NIH TB List Sorting (working memory) & $101.57(14.69)$ & $101.64(14.65)$ & $97.44(16.43)$ & $0.01^{*}$ \\
\hline Maternal age in years at birth & $29.73(6.05)$ & $29.72(6.04)$ & $30.44(6.34)$ & 0.20 \\
\hline Paternal age in years at birth & $31.98(6.90)$ & $31.95(6.89)$ & $33.28(7.08)$ & $0.04^{*}$ \\
\hline Categorical & Count (\%) & Count (\%) & Count (\%) & $x^{2}$ \\
\hline Male sex & $4778(52.33)$ & 4647 (51.75) & $131(86.75)$ & $6.49 \times 10^{-19^{\star \star \star}}$ \\
\hline White race & $6186(67.76)$ & $6084(67.76)$ & $102(67.55)$ & 1 \\
\hline Black race & $1185(12.98)$ & $1166(12.99)$ & 19 (12.58) & 1 \\
\hline "Other" race & $1715(18.78)$ & $1685(18.77)$ & $30(19.87)$ & 1 \\
\hline Latinx ethnicity & $1708(18.71)$ & $1686(18.78)$ & $22(14.57)$ & 0.48 \\
\hline Family history of psychosis & $957(10.48)$ & $937(10.44)$ & $20(13.25)$ & 0.49 \\
\hline History of speech delay & $1535(16.81)$ & 1445 (16.09) & $90(59.60)$ & $4.73 \times 10^{-32^{\star \star \star}}$ \\
\hline
\end{tabular}

Note: $M=$ mean; $S D=$ standard deviation; $p$-values are Benjamini-Hochberg corrected.

$* p<0.05$

** $p<0.01$

*** $p<0.001$ 
medRxiv preprint doi: https://doi.org/10.1101/2020.02.07.20021170; this version posted February 11, 2020. The copyright holder for this preprint (which was not certified by peer review) is the author/funder, who has granted medRxiv a license to display the preprint in perpetuity. It is made available under a CC-BY-NC-ND 4.0 International license.

Table 2

Predictors of continuous $P Q-B C$ distress score

\begin{tabular}{|c|c|c|c|c|c|c|}
\hline \multirow[t]{2}{*}{ Predictor } & \multirow[t]{2}{*}{$\beta$} & \multicolumn{2}{|c|}{$95 \% \mathrm{Cl}$} & \multirow[t]{2}{*}{$S E$} & \multirow[t]{2}{*}{$t$} & \multirow[t]{2}{*}{$p$} \\
\hline & & Lower & Upper & & & \\
\hline ASD & 2.46 & 1.32 & 3.60 & 0.58 & 4.23 & $2.31 \times 10^{-5^{\star \star \star}}$ \\
\hline Family history of psychosis & 1.05 & 0.56 & 1.53 & 0.25 & 4.20 & $2.76 \times 10^{-5^{\star \star \star}}$ \\
\hline Latinx ethnicity & 0.99 & 0.52 & 1.45 & 0.24 & 4.19 & $2.86 \times 10^{-5^{\star \star \star}}$ \\
\hline Black race & 0.89 & 0.30 & 1.48 & 0.30 & 2.95 & $3.17 \times 10^{-3^{\star \star}}$ \\
\hline Trauma index score & 0.55 & 0.26 & 0.85 & 0.15 & 3.68 & $2.30 \times 10^{-4^{\star \star \star}}$ \\
\hline Male sex & 0.13 & -0.17 & 0.42 & 0.15 & 0.84 & 0.40 \\
\hline NIH TB Pattern Comparison (processing spe $\epsilon$ & -0.24 & -0.56 & 0.08 & 0.16 & -1.45 & 0.15 \\
\hline NIH TB Card Sort (executive function) & -0.25 & -0.58 & 0.07 & 0.17 & -1.52 & 0.13 \\
\hline White race & -0.37 & -0.78 & 0.03 & 0.21 & -1.80 & 0.07 \\
\hline Age & -0.75 & -1.05 & -0.46 & 0.15 & -5.01 & $5.43 \times 10^{-7^{\star \star \star}}$ \\
\hline NIH TB List Sorting (working memory) & -1.12 & -1.43 & -0.81 & 0.16 & -7.11 & $1.29 \times 10^{-12^{\star \star \star}}$ \\
\hline Income-to-needs ratio & -1.23 & -1.58 & -0.89 & 0.18 & -7.02 & $2.34 \times 10^{-12^{\star \star \star}}$ \\
\hline (Intercept) & 3.25 & 2.55 & 3.95 & 0.36 & 9.07 & $1.68 \times 10^{-11^{* \star \star}}$ \\
\hline
\end{tabular}

Note: $95 \% \mathrm{Cl}=95 \%$ Wald confidence interval; $p$-values estimated via Satterthwaite's approximation.

${ }^{*}: p<0.05$
$* *$
$: p<0.01$
${ }^{* \star}: p<0.001$ 
medRxiv preprint doi: https://doi.org/10.1101/2020.02.07.20021170; this version posted February 11, 2020. The copyright holder for this preprint (which was not certified by peer review) is the author/funder, who has granted medRxiv a license to display the preprint in perpetuity. It is made available under a CC-BY-NC-ND 4.0 International license.

Table 3

Predictors of $P Q-B C$ distress score above $2 S D$ cutoff

\begin{tabular}{|c|c|c|c|c|c|c|}
\hline \multirow[t]{2}{*}{ Predictor } & \multirow[t]{2}{*}{$\beta$} & \multicolumn{2}{|c|}{$95 \% \mathrm{Cl}$} & \multirow[t]{2}{*}{$S E$} & \multirow[t]{2}{*}{$z$} & \multirow[t]{2}{*}{$p$} \\
\hline & & Lower & Upper & & & \\
\hline ASD & 3.18 & 1.77 & 5.69 & 0.94 & 3.88 & $1.03 \times 10^{-4^{\star \star \star}}$ \\
\hline Latinx ethnicity & 1.69 & 1.26 & 2.26 & 0.25 & 3.48 & $4.92 \times 10^{-4^{\star \star \star}}$ \\
\hline Family history of psychosis & 1.68 & 1.25 & 2.25 & 0.25 & 3.43 & $6.07 \times 10^{-4^{\star \star \star}}$ \\
\hline Black race & 1.24 & 0.87 & 1.76 & 0.22 & 1.17 & 0.24 \\
\hline Trauma index score & 1.20 & 1.01 & 1.44 & 0.11 & 2.03 & $0.04^{*}$ \\
\hline NIH TB Card Sort (executive function) & 0.96 & 0.75 & 1.23 & 0.12 & -0.31 & 0.76 \\
\hline Male sex & 0.96 & 0.78 & 1.19 & 0.10 & -0.38 & 0.71 \\
\hline NIH TB Pattern Comparison (processing spe $\epsilon$ & 0.88 & 0.70 & 1.11 & 0.10 & -1.10 & 0.27 \\
\hline White race & 0.79 & 0.60 & 1.04 & 0.11 & -1.67 & 0.09 \\
\hline Age & 0.75 & 0.60 & 0.94 & 0.08 & -2.51 & $0.01^{*}$ \\
\hline NIH TB List Sorting (working memory) & 0.51 & 0.40 & 0.65 & 0.06 & -5.56 & $2.70 \times 10^{-8^{\star \star \star}}$ \\
\hline Income-to-needs ratio & 0.49 & 0.38 & 0.64 & 0.07 & -5.28 & $1.32 \times 10^{-7^{\star \star \star}}$ \\
\hline (Intercept) & 0.02 & 0.01 & 0.04 & 0.01 & -14.34 & $1.23 \times 10^{-46^{\star \star \star}}$ \\
\hline
\end{tabular}

Note: $95 \% \mathrm{Cl}=95 \%$ Wald confidence interval; $p$-values estimated via Satterthwaite's approximation.

*: $p<0.05$

** $: p<0.01$

*** $p<0.001$ 
medRxiv preprint doi: https://doi.org/10.1101/2020.02.07.20021170; this version posted February 11, 2020. The copyright holder for this preprint (which was not certified by peer review) is the author/funder, who has granted medRxiv a license to display the preprint in perpetuity.

It is made available under a CC-BY-NC-ND 4.0 International license .

\section{Table S1}

Missingness by variable

\begin{tabular}{lrrr}
\hline Variable & $n$ missing & $n$ complete $\%$ complete \\
\hline ASD & 39 & 11836 & 99.67 \\
Age & 0 & 11875 & 100 \\
PQ-BC total score & 16 & 11859 & 99.87 \\
PQ-BC distress score & 16 & 11859 & 99.87 \\
Income-to-needs ratio & 1211 & 10664 & 89.80 \\
Trauma index score & 414 & 11461 & 96.51 \\
NIH TB Card Sort (executive function) & 162 & 11713 & 98.64 \\
NIH TB Pattern Comparison (processing spe & 181 & 11694 & 98.48 \\
NIH TB List Sorting (working memory) & 206 & 11669 & 98.27 \\
Maternal age in years at birth & 264 & 11611 & 97.78 \\
Paternal age in years at birth & 643 & 11232 & 94.59 \\
Sex & 3 & 11872 & 99.97 \\
Race & 0 & 11875 & 100 \\
Ethnicity & 13 & 11862 & 99.89 \\
Family history of psychosis & 450 & 11425 & 96.21 \\
History of speech delay & 6 & 11869 & 99.95 \\
\hline
\end{tabular}

Note: Numbers for "ASD," "family history of psychosis" and "speech delay" denote whether data was present, not whether variable was positive. 
medRxiv preprint doi: https://doi.org/10.1101/2020.02.07.20021170; this version posted February 11, 2020. The copyright holder for this preprint (which was not certified by peer review) is the author/funder, who has granted medRxiv a license to display the preprint in perpetuity.

It is made available under a CC-BY-NC-ND 4.0 International license .

Table S2

Item-level $P Q-B C$ distress score comparisons

\begin{tabular}{ll}
\hline \multicolumn{1}{c}{ PQ-BC item } \\
\cline { 1 - 2 } 1 & $\begin{array}{l}\text { Question ("In the past month ... ") } \\
\text { your bedroom, or other rooms in your } \\
\text { home, your classroom, or school } \\
\text { yard, suddenly seem weird, strange or } \\
\text { confusing to you, like not the real }\end{array}$
\end{tabular}
world?

2 Did you hear strange sounds that you never noticed before, like banging,

$0.30(0.88)$

$0.50(1.20)$

$0.08(0.47)$

$0.04(0.36)$

$0.05(0.27)$

0.03

0.86 unusual powers, like you could make things happen by magic, or that you could magically know what was inside another person's mind, or magically know what was going to happen in the future when other people could not?

5 Did you feel that someone else, who is not you, has taken control over the private, personal thoughts or ideas inside your head?

6 Did you suddenly find it hard to figure out how to say something quickly and easily so that other people would understand what you meant?

$7 \quad$ Did you ever feel very certain that you have very special abilities or magical talents that other people do not have? Did you suddenly feel that you could not trust other people because they seemed to be watching you or talking about you in an unfriendly way?

$9 \quad$ Did your skin or just beneath your skin suddenly start feeling strange, like bugs crawling?

10 Did you lose concentration because you noticed sounds in the distance that you usually don't hear?

11 Although you could not see anything or anyone, did you suddenly start to feel that an invisible energy, creature, or some person was around you?

12 Did you start to worry at times that your mind was trying to trick you or was not working right?

13 Did you feel that the world is not real, you are not real, or that you are dead?

14 Did you feel confused because something you experienced didn't seem real, or it seemed imaginary to you?

15 Did you honestly believe in things that other people would say are unusual or weird? $\begin{array}{llll}0.11(0.61) & 0.21(0.87) & 4.23 & 0.07\end{array}$

$0.16(0.64)$

$0.21(0.74)$

1.01

0.37

$0.03(0.30)$

$0.07(0.50)$

2.40

0.16

$0.45(1.11)$

$0.60(1.37)$

$0.26(0.88)$

$0.34(1.03)$

1.13

0.36

$0.22(0.81)$

$0.41(1.14)$

8.00

0.02

$0.38(1.09)$

$0.68(1.49)$

10.80

$0.01^{\star}$

$0.20(0.81)$

$0.36(1.12)$

6.27

$0.03^{\star}$

$0.09(0.57)$

$0.18(0.83)$

3.66

0.09

$0.11(0.58)$

$0.22(0.84)$

5.20

0.04

$0.12(0.63)$

$0.29(1.01)$

11.29

$0.01^{\star}$

\begin{tabular}{cccc}
$M(S D)$ & $F$ & $p$ \\
\hline No ASD $(n=8979)$ & ASD $(n=151)$ & & \\
$0.08(0.50)$ & $0.19(0.77)$ & 6.38 & $0.03^{*}$
\end{tabular}


medRxiv preprint doi: https://doi.org/10.1101/2020.02.07.20021170; this version posted February 11, 2020. The copyright holder for this preprint

(which was not certified by peer review) is the author/funder, who has granted medRxiv a license to display the preprint in perpetuity.

It is made available under a CC-BY-NC-ND 4.0 International license.

16

Did you feel that parts of your body had suddenly changed or worked differently than before, like your legs had suddenly turned to something else, or your nose could suddenly smell things you'd never

17 Did you feel that sometimes your actuallv smelled before? thoughts were so strong you could almost hear them, as if another person, NOT you, spoke them?

18 Did you feel that other people might want something bad to happen to you or that you could not trust other people?

19 Did you suddenly start to see unusual things that you never saw before, like flashes, flames, blinding light, or shapes floating in front of you?

20 Did you suddenly start to be able to see things that other people could not see, or they did not seem to see?

21 Did you suddenly start to notice that people sometimes had a hard time understanding what you were saying, even though they used to understand you well?

$$
0.06(0.43)
$$

$0.07(0.34)$

0.09

0.84

$0.09(0.53)$

$0.21(0.81)$

7.21

$0.02^{*}$

$0.28(0.95)$

0.49 (1.28)

7.08

$0.02^{*}$

$0.17(0.76)$

$0.28(0.95)$

3.06

0.12

$0.11(0.63)$

$0.24(0.88)$

6.04

$0.03^{*}$

$0.13(0.62)$

$0.32(1.06)$

13.65

$4.65 \times 10^{-3^{* \star}}$ Note: $M=$
${ }^{*}: p<0.05$
${ }^{* *}: p<0.01$ 
medRxiv preprint doi: https://doi.org/10.1101/2020.02.07.20021170; this version posted February 11, 2020. The copyright holder for this preprint (which was not certified by peer review) is the author/funder, who has granted medRxiv a license to display the preprint in perpetuity.

It is made available under a CC-BY-NC-ND 4.0 International license.

Table S3

Predictors of parent-reported ASD

\begin{tabular}{|c|c|c|c|c|c|c|}
\hline \multirow[t]{2}{*}{ Predictor } & \multirow[t]{2}{*}{$\beta$} & \multicolumn{2}{|c|}{$\underline{95 \% \mathrm{Cl}}$} & \multirow[t]{2}{*}{$S E$} & \multirow[t]{2}{*}{$z$} & \multirow[t]{2}{*}{$p$} \\
\hline & & Lower & Upper & & & \\
\hline History of speech delay & 6.09 & 4.34 & 8.53 & 0.17 & 10.49 & $<2 \times 10^{-16^{\star \star \star}}$ \\
\hline Male sex & 4.48 & 2.77 & 7.25 & 0.25 & 6.10 & $1.06 \times 10^{-9 \star \star \star}$ \\
\hline Paternal age & 1.64 & 1.02 & 2.64 & 0.24 & 2.04 & 0.04 \\
\hline Age & 1.25 & 0.90 & 1.72 & 0.16 & 1.33 & 0.18 \\
\hline White race & 0.92 & 0.60 & 1.42 & 0.22 & 0.37 & 0.71 \\
\hline Maternal age & 0.88 & 0.52 & 1.50 & 0.27 & 0.46 & 0.64 \\
\hline Black race & 0.87 & 0.45 & 1.68 & 0.33 & 0.41 & 0.68 \\
\hline Income-to-needs ratio & 0.76 & 0.51 & 1.13 & 0.20 & 1.37 & 0.17 \\
\hline Latinx ethnicity & 0.64 & 0.38 & 1.07 & 0.27 & 1.69 & 0.09 \\
\hline (Intercept) & $3.39 \times 10^{-3}$ & $1.85 \times 10^{-3}$ & 0.01 & 0.31 & 18.38 & $<2 \times 10^{-16^{\star \star \star}}$ \\
\hline
\end{tabular}

Note: $95 \% \mathrm{Cl}=95 \%$ Wald confidence interval.

* $p<0.05$

** $p<0.01$

$*: p<0.001$ 